\title{
Polimorfismos funcionales del gen VEGF no están asociados con degeneración macular relacionada con la edad en población mexicana
}

\section{Functional polymorphisms in the VEGF gene are not associated with age-related macular degeneration in a Mexican population}

\author{
Ramses Rosales-Diaz' ${ }^{1}$, Arturo Santos-Garcia ${ }^{2,3}$, Jose Navarro-Partida ${ }^{2,3}$, Veronica Benites-Godinez ${ }^{4}$, \\ Daniel Díaz-Palomera ${ }^{5}$ y Adolfo D. Rodríguez-Carrizalez ${ }^{6 *}$ \\ ${ }^{1}$ Unidad Académica de Medicina, Universidad Autónoma de Nayarit, Tepic, Nay.; ${ }^{2}$ Tecnológico de Monterrey, Escuela de Medicina y Ciencias de \\ la Salud, Zapopan, Jal.; ${ }^{3}$ Centro de Retina Médica y Quirúrgica, Centro Médico Puerta de Hierro. Zapopan, Jal.; ${ }^{4}$ Instituto Mexicano del Seguro \\ Social, Tepic, Nay.; ${ }^{5}$ Instituto de Biología Molecular en Medicina y Terapia Génica, Universidad de Guadalajara, Guadalajara, Jal.; ${ }^{6}$ Instituto de \\ Terapéutica Experimental y Clínica, Departamento de Fisiología, Centro Universitario de Ciencias de la Salud, Universidad de Guadalajara, \\ Guadalajara, Jal. México
}

\section{Resumen}

Introducción: La degeneración macular relacionada con la edad (DMRE) exudativa está asociada con la expresión del factor de crecimiento vascular endotelial (VEGF). Varios polimorfismos de un solo nucleótido (SNP) en el gen VEGF están asociados con diferentes patrones de expresión de esta citocina (polimorfismos funcionales). En este estudio consideramos 4 SNP que están ubicados en la región promotora (rs699947 A/C, rs833061 C/T, rs1005230 C/T y rs1570360 A/G) y dos en la región 5’ no traducida (rs2010963 C/G y rs25648 C/T) que tienen efecto sobre la regulación de la transcripción. Objetivo: Asociar SNP funcionales con la DMRE exudativa en población mexicana. Métodos: A través de reacción en cadena de la polimerasa con ensayos de discriminación alélica con sondas Taqman ${ }^{\circledR}$, los polimorfismos fueron genotipados. Las frecuencias genotípicas se compararon utilizando todos los modelos de herencia. Resultados: Los SNP fueron genotipados en 105 pacientes diagnosticados con DMRE exudativa (61 mujeres y 44 hombres, edad promedio $74.28 \pm 8.32$ años) y en 102 sujetos control sanos (61 mujeres y 41 hombres, edad promedio $67.41 \pm 5.78$ años). Los genotipos en ambos grupos estaban en el equilibrio de Hardy-Weinberg. En cualquiera de los SNP analizados, la frecuencia de alelos menores fue diferente entre los grupos, con significación estadística. Los genotipos no mostraron diferencias estadísticamente significativas entre la DMRE exudativa y los grupos control ( $p>0.05)$. Conclusión: No se encontró asociación entre los SNP estudiados y la presencia de DMRE exudativa en la población mexicana analizada. Consideramos necesario un análisis adicional en muestras más grandes para confirmar estos hallazgos.

Palabras clave: Polimorfismos. Degeneración macular asociada con la edad. Factor de crecimiento vascular endotelial.

\begin{abstract}
Introduction: The wet form of age-related macular degeneration (WAMD) is related to the expression of vascular endothelial growth factor (VEGF). Several single nucleotide polymorphisms (SNPS) in the VEGF gene are associated with different ex-
\end{abstract}

Correspondencia:

*Adolfo Daniel Rodríguez-Carrizalez

Instituto de Terapéutica Experimental y Clínica

Col. Independencia

Fecha de recepción: 23-08-2019

Fecha de aceptación: 17-02-2020

C.P. 44340, Guadalajara, Jal., México

E-mail: adolfo.rodriguez@academicos.udg.mx DOI: 10.24875/RMO.M20000116
Disponible en internet: 01-05-2020 Rev Mex Oftalmol. 2020;94(3):124-130

www.rmo.com.mx (http://creativecommons.org/licenses/by-nc-nd/4.0/). 
pression patterns of this cytokine (functional polymorphisms). In this study, we considered 4 SNPs that are located in the promoter region ( $r s 699947 \mathrm{~A} / \mathrm{C}, \mathrm{rs} 833061 \mathrm{C} / \mathrm{T}$, rs $1005230 \mathrm{C} / \mathrm{T}$ and rs1570360 A/G) and two in the 5' untranslated region (rs2010963 C/G and rs25648 C/T) that have an effect on transcriptional regulation. Objective: To associate functional SNPS with WAMD in a Mexican population. Methods: Through allelic discrimination assays, consisting in polymerase chain reactions with Taqman ${ }^{\circledR}$ probes, the polymorphisms were genotyped. Genotype frequencies were compared using all inheritance models. Results: SNPS were genotyped in 105 patients diagnosed with WAMD (61 women and 44 men, mean age $74.28 \pm 8.32$ years), and in 102 healthy control subjects (61 women and 41 men, mean age $67.41 \pm 5.78$ years). Genotypes in both groups were in Hardy-Weinberg equilibrium. In all SNPs analyzed, the minor allele frequency was statistically significant different between the groups. Genotypes were not statistically significant different between the WAMD and the control groups ( $p>0.05$ ). Conclusion: No association was observed between the studied SNPs and the presence of WAMD in the analyzed Mexican population. We consider that further analysis in larger samples is necessary to confirm these findings.

Key words: Polymorphisms. Age-related macular degeneration. Vascular endothelial growth factor.

\section{Introducción}

La degeneración macular relacionada con la edad (DMRE) es una enfermedad neurodegenerativa de la retina, caracterizada por una disminución progresiva de la visión central. Representa la principal causa de pérdida visual en personas mayores de 50 años ${ }^{1}$.

La DMRE puede presentarse en dos formas clínicas bien diferenciadas, cada una con diferentes características patológicas. La DMRE atrófica no exudativa se caracteriza por una atrofia progresiva del epitelio pigmentario de la retina, de los fotorreceptores y de la coriocapilar. Por el contrario, en la forma neovascular, exudativa o húmeda de la DMRE (DMRE-NV), se ha desarrollado neovascularización coroidea (NVC) y tejido fibroso neovascular subretiniano debido a la fuga de líquido y lípidos dentro de la retina ${ }^{1,2}$.

La DMRE es una enfermedad multifactorial. Algunos de los factores de riesgo asociados son la edad, el tabaquismo, la exposición a la luz solar, la dieta y la genética. Diferentes estudios han demostrado el importante papel que juegan los factores genéticos en la fisiopatología de la $\mathrm{DMRE}^{3-5}$. Se han involucrado varios genes en diferentes vías biológicas, como las vías inmunes y del complemento ( $\mathrm{CFH}, \mathrm{C2} / \mathrm{CFB}, \mathrm{CFI}, \mathrm{C} 3, \mathrm{y}$ C9), el transporte de lípidos (APOE, LIPC, CETP y BAIAP2L2), la remodelación de la matriz extracelular (COL8A1, COL10A1, TIMP3, ADAMTS9, TGFBR1, HTRA1 y B3GALTL) y la angiogénesis (VEGFA, TGFBR1, y ADAMTS9) ${ }^{6}$. El factor de crecimiento vascular endotelial (VEGF) es una de las principales citocinas involucradas en las vías de angiogénesis y NVC, presente en la DMRE-NV.

La familia de citocinas del VEGF incluye: VEGF-A, VEGF-B, VEGF-C, VEGF-D, VEGF-E y factor de crecimiento placentario (PGF). Estos factores son críticos para el desarrollo y mantenimiento de la función vascular normal, y afectan directamente la proliferación, supervivencia y migración de las células endoteliales. Respectivamente, cada isoforma del VEGF puede tener una función angiogénica o antiangiogénica. Con respecto al efecto angiogénico, VEGF-A es el mediador principal. La angiogénesis ocurre como resultado de varios estímulos, como la hipoxia tisular, el estrés por cizallamiento y el desarrollo de células tumorales; en consecuencia, mejorando la perfusión sanguínea ${ }^{7-9}$. Más recientemente, en diferentes poblaciones, se ha observado previamente la asociación entre ciertos polimorfismos del VEGF y su papel como factores de riesgo genéticos en la DMRE-NV ${ }^{10,11}$. Sin embargo, los resultados son controvertidos. Por ejemplo, el polimorfismo con rs833061 ubicado en la región promotora del gen VEGF, que se ha relacionado con una mayor actividad promotora ${ }^{12,13}$ y con incremento en el riesgo de presentar DMRE-NV en población étnica Tujia de China y en población polaca ${ }^{14}$. Por el contrario, otros estudios no han encontrado correlación con la susceptibilidad a la DMRE-NV en las poblaciones chinas de Taiwán, Han y de España ${ }^{15-17}$.

Existen más de 100 polimorfismos de un solo nucleótido (SNP) identificados en el gen VEGF hasta la fecha (http://www.ncbi.nlm.nih.gov/SNP). Muchos de ellos están asociados con diferentes patrones de expresión de esta citocina, y comúnmente se denominan polimorfismos funcionales. En este estudio, consideramos 4 SNP funcionales en el gen VEGF que se encuentran en la región promotora (rs699947 A/C, rs833061 C/T, rs1005230 C/T y rs $1570360 \mathrm{~A} / \mathrm{G}$ ) y dos en la región 5' no traducida (rs2010963 C/G y rs25648 C/T), que se han relacionado positivamente con la transcripción del gen VEGF ${ }^{15-22}$. Todo esto, con el propósito de establecer un patrón genético de una población mexicana, que podría determinar el riesgo de desarrollo de DMRE exudativa. 


\section{Pacientes y métodos}

\section{Pacientes}

Un total de 105 pacientes que no estaban relacionados (44 hombres y 61 mujeres; edad, $74.28 \pm 8.32$ años) se incluyeron en el grupo de casos. Fueron diagnosticados con DMRE-NV en el Centro Médico Nacional de Occidente, Instituto Mexicano del Seguro Social, Jal., México. Los criterios para clasificar a un paciente como caso fueron: examen oftalmológico con datos de DMRE-NV, líquido subretiniano, hemorragias y lípidos asociados con una membrana macular de color gris-marrón y hallazgos en la angiografía con fluorescencia retiniana de NVC. Los pacientes fueron excluidos si tenían diagnóstico de NVC secundaria a trauma o no asociada con la edad. Un total de 41 hombres y 61 mujeres con una edad promedio de $67.41 \pm$ 5.78 años se incluyeron en el grupo control. Los criterios de inclusión para el grupo control fueron: examen oftalmológico sin datos sugestivos de DMRE-NV y edad superior a 60 años. El estudio fue aprobado por el comité de ética del Centro de Retina Médica y Quirúrgica S.C., y cumplió los principios de la Declaración de Helsinki. Se obtuvo el consentimiento informado para el cribado genético de todos los sujetos (casos y controles).

\section{Preparación de muestras y genotipado}

Los SNP objetivo del gen VEGF-A se seleccionaron con base en su fuerte asociación con la DMRE-NV. Los SNP seleccionados se ubican en regiones no codificantes y promotoras del gen VEGF-A (locus 6p21.1): rs2010963 C/G y rs25648 C/T dentro de la región $5^{\prime}$ no traducida y rs833061 C/T, rs699947 A/C, rs1570360 A/G y rs1005230 C/T, dentro de la región promotora. Las ubicaciones de estos polimorfismos son 5398, $6025,4534,34374878$ y 3544 , respectivamente (Assembly; GRCh38.p7, RefSeqGene, NG_008732.1; Gene ID, VEGFA 7422).

El ADN genómico se extrajo de sangre completa congelada purificada con el kit para $A D N$ en sangre QIAamp de Qiagen (Qiagen, Valencia, CA, EE.UU.). EI genotipado de los SNP objetivo se realizó utilizando ensayos de genotipado de SNP TaqMan ${ }^{\circledR}$ validados (TaqMan: Applied Biosystems, Inc. [ABI], Foster City, CA). Los SNP se amplificaron utilizando reacción en cadena de la polimerasa (PCR) en tiempo real con 0.5 $\mu \mathrm{M}$ de cebadores y Master Mix para genotipado de TaqMan (TaqMan: Applied Biosystems, Inc. [ABI],
Foster City, CA) con $30 \mathrm{ng}$ de ADN templado en la mezcla de amplificación $(25 \mu \mathrm{l})$. La señal de fluorescencia de la sonda se detectó con el sistema de PCR en tiempo real StepOnePlus ${ }^{\mathrm{TM}}$ (Applied Biosystems, Inc. $[A B I]$, Carlsbad, CA).

\section{Cálculo del tamaño de la muestra y análisis estadísticos}

La prevalencia de la DMRE aumenta con la edad y está influenciada por el origen étnico. La prevalencia puede ser tan baja como $2.5 \%$ en adultos blancos mayores de 50 años, hasta el $14 \%$ en adultos blancos mayores de 80 años. Por lo tanto, considerando un modelo genético dominante y asumiendo un $5 \%$ de frecuencia de alelos menores, $5 \%$ de prevalencia de la enfermedad, proporción 1:1 de casos y controles, y $5 \%$ de error tipo I y una OR esperada de 2 para el análisis de marcadores únicos, el número mínimo de casos requeridos para lograr un poder del $80 \%$ era de 90 . Se utilizó el software estadístico IBM SPSS (versión 21.0, SPSS, Inc., Chicago, IL) para los análisis estadísticos. El equilibrio de Hardy-Weinberg se analizó utilizando frecuencias de genes obtenidas mediante un conteo simple de genes y la prueba de Chi cuadrado con corrección de Yates para comparar los valores observados y esperados. Las diferencias en las frecuencias de genotipo entre los casos y los controles se analizaron mediante la prueba exacta de Fisher o mediante Chi cuadrado. Los haplotipos inferidos y el LD (desequilibrio del ligamiento) cuantificados entre los loci bialélicos se calcularon utilizando el software en línea SNPStats (http://bioinfo.iconcologia.net/SNPStats). La importancia de una asociación se determinó mediante análisis de tablas de contingencia utilizando Chi cuadrado o la prueba exacta de Fisher. La significancia se definió como un valor de $p$ menor de 0.05 .

\section{Resultados}

\section{Frecuencias de alelos y genotipos}

Todos los SNP se genotipificaron en todos los sujetos; las frecuencias se encontraron en equilibrio de Hardy-Weinberg en ambos grupos. La diferencia entre las frecuencias alélicas no fue estadísticamente significativa en ninguno de los SNP analizados (Tabla 1).

Las frecuencias genotipificadas se compararon aplicando los modelos codominante, dominante y recesivo. Ninguno de los modelos de herencia mostró una asociación estadísticamente significativa en el grupo 
Tabla 1. Frecuencia de alelos de SNP en los grupos control y con DMRE

\begin{tabular}{|c|c|c|c|c|c|}
\hline \multirow{2}{*}{$\begin{array}{l}\text { SNP/ } \\
\text { alelos }\end{array}$} & & DMRE & Control & \multirow[t]{2}{*}{ OR (IC 95\%) } & \multirow[t]{2}{*}{$\mathbf{p}$} \\
\hline & & $n=210$ & $\mathrm{n}=\mathbf{2 0 4}$ & & \\
\hline $\begin{array}{l}\text { rs } 1570360 \\
\text { (E) }\end{array}$ & $A / G$ & $49(0.23)$ & $45(0.22)$ & $\begin{array}{c}1.0754 \\
(0.678-1.703)\end{array}$ & 0.75 \\
\hline $\begin{array}{l}\text { rs833061 } \\
\text { (H) }\end{array}$ & $\mathrm{C} / \mathrm{T}$ & $90(0.43)$ & $84(0.41)$ & $\begin{array}{c}1.0714 \\
(0.725-1.583)\end{array}$ & 0.72 \\
\hline $\begin{array}{l}\text { rs } 2010963 \\
\text { (F) }\end{array}$ & $\mathrm{C} / \mathrm{G}$ & $82(0.39)$ & $78(0.38)$ & $\begin{array}{c}1.0349 \\
(0.696-1.537)\end{array}$ & 0.86 \\
\hline $\begin{array}{l}\text { rs } 25648 \\
\text { (K) }\end{array}$ & $\mathrm{C} / \mathrm{T}$ & $176(0.84)$ & $164(0.8)$ & $\begin{array}{c}1.2626 \\
(0.762-2.090)\end{array}$ & 0.36 \\
\hline $\begin{array}{l}\text { rs699947 } \\
\text { (I) }\end{array}$ & $A / C$ & $85(0.4)$ & $86(0.42)$ & $\begin{array}{c}0.933 \\
(0.630-1.379)\end{array}$ & 0.72 \\
\hline $\begin{array}{l}\text { rs1005230 } \\
\text { (J) }\end{array}$ & $\mathrm{C} / \mathrm{T}$ & $125(0.6)$ & $\begin{array}{c}121 \\
(0.59)\end{array}$ & $\begin{array}{c}1.0088 \\
(0.681-1.493)\end{array}$ & 1 \\
\hline
\end{tabular}

DMRE: degeneración macular relacionada con la edad; IC: intervalo de confianza; OR: odds ratio; SNP: polimorfismo de un solo nucleótido. Fuente: RefSeqGene, NG_008732.1; Gene ID, VEGFA (7422), https://www.ncbi.nlm.nih.gov/refseq/rsg/

de DMRE-NV en comparación con el grupo control (Tabla 2).

\section{Análisis de haplotipos}

Todos los SNP etiqueta se ubicaron en un bloque de haplotipos, y la magnitud del LD entre los loci bialélicos fue muy alta, con una D' pareada $=0.8205$. Las frecuencias de haplotipo de los SNP analizados se muestran en la tabla 3. Los SNP etiqueta no fueron estadísticamente significativos.

\section{Discusión}

El gen VEGF-A humano se encuentra en la región cromosómica $6 \mathrm{p} 21.1$. Se generan varias isoformas mediante splicing alternativo que produce proteínas de 121, 145, 162, 165, 183, 189 y 206 aminoácidos con diferencias en sus propiedades. Cada tejido expresa diferentes proporciones de las isoformas de VEGF dependiendo de sus necesidades específicas. La isoforma VEGF-165 es la que se ha observado con más frecuencia en el ojo humano $23-25$.

Su papel en la NVC se ha demostrado claramente 9. Por ejemplo, se ha demostrado un aumento de la expresión de VEGF en el ojo en modelos animales ${ }^{26-29}$.

Se están investigando varios factores genéticos relacionados con el control de la expresión de VEGF por su papel en la patogénesis de DMRE-NV. Se observó que los factores potenciadores de los miocitos 2 (MEF2) activan la expresión transcripcional del VEGF a través de ligando tipo Delta 4 (DII4) durante la angiogénesis ${ }^{30}$. Otro estudio mostró que un coactivador transcripcional (PGC-1 $\alpha$ ) es capaz de regular la expresión de VEGF-A en la retina ${ }^{31}$. Sin embargo, los vínculos moleculares entre las señales proangiogénicas y la fisiopatología de la DMRE-NV son aún limitados.

Estudios previos sobre la asociación de SNP del gen VEGF-A con la DMRE-NV mostraron resultados divergentes en diferentes poblaciones. El polimorfismo con rs833061 ubicado en la región promotora del gen $V E G F$, que se ha relacionado con un incremento de la actividad promotora ${ }^{12,13}$, ha sido analizado en estudios previos que sugirieron que la DMRE-NV tiene una correlación débil con este SNP en un grupo de chinos Tujia y en una población polaca ${ }^{14}$. Por el contrario, otros estudios no han encontrado correlación con la susceptibilidad a la DMRE-NV en las poblaciones chinas de Taiwán, Han y de España ${ }^{15-17}$.

El polimorfismo rs699947 ubicado en la región promotora del gen VEGF ha mostrado asociación con una mayor producción de VEGF ${ }^{18}$. En un estudio de cohorte en población coreana, la presencia del alelo principal se asoció con una buena respuesta visual a la terapia anti-VEGF debido a su menor producción de VEGF ${ }^{32}$. Por un lado, varios estudios no han mostrado asociación entre SNP y la DMRE-NV en la población de Países Bajos, China de Taiwán, Finlandia, Japón, España y Turquía ${ }^{15,17,19-22}$. Por otro lado, en una población de Pakistán se ha demostrado una asociación de riesgo entre el polimorfismo y la morbilidad de la DMRE-NV ${ }^{33}$. Sin embargo, nuestros datos son consistentes con los hallazgos reportados en la mayoría de las poblaciones.

Aunque rs2010963 es un polimorfismo funcional asociado con niveles elevados de VEGF-A en suero y vítreo en la retina humana ${ }^{34,35}$, no se ha demostrado una asociación farmacogenética con la respuesta a los agentes anti-VEGF en diferentes poblaciones ${ }^{36,37}$. Además, la asociación entre este polimorfismo y la DMRE-NV no se ha demostrado claramente, existen discrepancias entre poblaciones como la china, la japonesa y la taiwanesa, donde no existe una asociación significativa, y la polaca, donde sí se ha observado dicha asociación ${ }^{14-16,21 .}$

El polimorfismo rs25648 se ha estudiado en patologías en las que la angiogénesis juega un papel importante, como el cáncer de vejiga, que muestra un aumento significativo en el riesgo de desarrollarlo ${ }^{38}$. Sin embargo, en este estudio no se demostró que haya 
Tabla 2. Frecuencia de genotipos en polimorfismos de un solo nucleótido del VEGF

\begin{tabular}{|c|c|c|c|c|c|c|c|}
\hline \multirow[t]{2}{*}{ Posición del gen } & \multirow[t]{2}{*}{ SNP } & \multirow[t]{2}{*}{ SNP posición } & \multirow[t]{2}{*}{ Ubicación del SNP } & \multirow[t]{2}{*}{ Genotipos } & DMRE & Control & \multirow[t]{2}{*}{ p } \\
\hline & & & & & $n=105$ & $\mathrm{n}=102$ & \\
\hline \multirow[t]{6}{*}{$6 \mathrm{p} 21.1$} & rs833061 & 4534 & región promotora & $\begin{array}{l}\mathrm{C} / \mathrm{T} \\
\mathrm{C} / \mathrm{C} \\
\mathrm{T} / \mathrm{T}\end{array}$ & $\begin{array}{l}54(0.51) \\
18(0.17) \\
33(0.31)\end{array}$ & $\begin{array}{l}48(0.47) \\
18(0.18) \\
36(0.35)\end{array}$ & 0.8 \\
\hline & rs699947 & 3437 & región promotora & $\begin{array}{l}A / C \\
A / A \\
C / C\end{array}$ & $\begin{array}{l}55(0.52) \\
15(0.14) \\
35(0.33)\end{array}$ & $\begin{array}{c}46(0.45) \\
20(0.2) \\
36(0.35)\end{array}$ & 0.47 \\
\hline & rs 1005230 & 3544 & región promotora & $\begin{array}{l}\mathrm{C} / \mathrm{T} \\
\mathrm{C} / \mathrm{C} \\
\mathrm{T} / \mathrm{T}\end{array}$ & $\begin{array}{l}55(0.52) \\
35(0.33) \\
15(0.14)\end{array}$ & $\begin{array}{l}47(0.46) \\
37(0.36) \\
18(0.18)\end{array}$ & 0.63 \\
\hline & rs 1570360 & 4878 & región promotora & $\begin{array}{l}A / G \\
A / A \\
G / G\end{array}$ & $\begin{array}{c}37(0.35) \\
6(0.06) \\
62(0.59)\end{array}$ & $\begin{array}{c}37(0.36) \\
4(0.04) \\
61(0.6)\end{array}$ & 0.83 \\
\hline & rs2010963 & 5398 & UTR 5' & $\begin{array}{l}\mathrm{C} / \mathrm{G} \\
\mathrm{C} / \mathrm{C} \\
\mathrm{G} / \mathrm{G}\end{array}$ & $\begin{array}{l}50(0.48) \\
16(0.15) \\
39(0.37)\end{array}$ & $\begin{array}{l}48(0.47) \\
15(0.15) \\
39(0.38)\end{array}$ & 0.98 \\
\hline & rs 25648 & 6025 & UTR 5' & $\begin{array}{l}\mathrm{C} / \mathrm{T} \\
\mathrm{C} / \mathrm{C} \\
\mathrm{T} / \mathrm{T}\end{array}$ & $\begin{array}{c}24(0.23) \\
76(0.72) \\
5(0.05)\end{array}$ & $\begin{array}{c}36(0.35) \\
64(0.63) \\
2(0.02)\end{array}$ & 0.096 \\
\hline
\end{tabular}

DMRE: degeneración macular relacionada con la edad; IC: intervalo de confianza; OR: odds ratio; SNP: polimorfismo de un solo nucleótido; UTR: región no traducida; VEGF: factor de crecimiento vascular endotelial.

Fuente: RefSeqGene, NG_008732.1; Gene ID, VEGFA (7422), https://www.ncbi.n/m.nih.gov/refseq/rsg/

Tabla 3. Frecuencias de haplotipos de los SNP etiqueta en el gen VEGF entre los sujetos con DMRE y control

\begin{tabular}{|c|c|c|c|c|c|c|c|c|c|c|}
\hline \multicolumn{6}{|c|}{ SNP etiqueta } & \multirow[t]{2}{*}{ Total } & \multirow[t]{2}{*}{ DMRE } & \multirow[t]{2}{*}{ Control } & \multirow[t]{2}{*}{ OR (IC 95\%) } & \multirow[t]{2}{*}{ p } \\
\hline rs1570360 & rs2010963 & rs833061 & rs699947 & rs1005230 & rs 25648 & & & & & \\
\hline G & C & $\mathrm{T}$ & C & C & C & 0.369 & 0.3905 & 0.3701 & 1 & - \\
\hline A & G & C & A & $\mathrm{T}$ & C & 0.2263 & 0.2227 & 0.2156 & $0.97(0.57-1.64)$ & 0.91 \\
\hline G & G & $\mathrm{T}$ & C & C & C & 0.187 & 0.171 & 0.1933 & $1.18(0.67-2.07)$ & 0.58 \\
\hline G & G & C & A & $\mathrm{T}$ & $\mathrm{T}$ & 0.1543 & 0.1375 & 0.1713 & $1.20(0.67-2.13)$ & 0.54 \\
\hline G & G & C & $A$ & $\mathrm{~T}$ & C & 0.0195 & 0.034 & 0.0048 & $0.16(0.02-1.43)$ & 0.1 \\
\hline G & G & C & C & C & C & 0.0121 & 0.02 & 0.0049 & $0.28(0.02-3.25)$ & 0.31 \\
\hline
\end{tabular}

DMRE: degeneración macular relacionada con la edad; SNP: polimorfismo de un solo nucleótido; VEGF: factor de crecimiento vascular endotelial.

una asociación con la DMRE-NV en nuestra población. Además, este polimorfismo en la DMRE-NV no se ha estudiado claramente. En un estudio que incluyó población caucásica, no se encontró una asociación ${ }^{39}$.

Existe información consistente sobre la correlación del SNP rs1570360 con enfermedades vasculares como el accidente cerebrovascular y la hipertensión $\mathrm{n}^{40,41}$ y su actividad, con mayor producción de VEGF-A ${ }^{13}$. En nuestra población, así como en la población japonesa y holandesa, no hay asociación entre el polimorfismo y la susceptibilidad de DMRE-NV ${ }^{19,21}$. Del mismo modo, no se ha encontrado ninguna asociación del polimorfismo rs1005230 con la DMRE-NV, ni se ha demostrado que mejore la respuesta al tratamiento anti-VEGF en pacientes con DMRE-NV ${ }^{42}$.

Hasta la fecha, todavía existe información contrastante sobre la asociación de polimorfismos del gen VEGF con la DMRE-NV en diferentes poblaciones. Aunque algunos estudios han encontrado una asociación de riesgo, en la población mexicana se demostró que no existe asociación con ninguno de los SNP estudiados. Sin embargo, estos hallazgos estadísticamente 
no significativos podrían estar relacionados con el tamaño de la muestra. De hecho, la principal limitación de nuestro estudio es el reducido número de sujetos incluidos. Por lo tanto, es necesario expandir la muestra para estimar con precisión las asociaciones de riesgo.

\section{Conclusión}

No se observó una asociación entre los SNP estudiados y la presencia de DMRE-NV en la población mexicana analizada. Estos hallazgos concuerdan con informes previos evaluados en poblaciones de los Países Bajos, Taiwán, China, Finlandia, Japón, España y Turquía. Consideramos que es necesario realizar análisis en muestras más grandes para confirmar estos hallazgos.

\section{Responsabilidades éticas}

Protección de personas y animales. Los autores declaran que los procedimientos seguidos se conformaron a las normas éticas del comité de experimentación humana responsable y de acuerdo con la Asociación Médica Mundial y la Declaración de Helsinki.

Confidencialidad de los datos. Los autores declaran que siguieron los protocolos de su centro de trabajo sobre la publicación de datos de pacientes.

Derecho a la privacidad y consentimiento informado. Los autores obtuvieron el consentimiento informado de los pacientes y/o sujetos referidos en el artículo. Este documento obra en poder del autor de correspondencia.

\section{Conflicto de intereses}

Los autores declaran no tener ningún conflicto de intereses. Los autores son los únicos responsables del contenido y la redacción de este artículo.

\section{Bibliografía}

1. Barchitta M, Maugeri A. Association between Vascular Endothelial Growth Factor Polymorphisms and Age-Related Macular Degeneration: An Updated Meta-Analysis. Dis Markers. 2016;2016:8486406.

2. Lim LS, Mitchell P, Seddon JM, Holz FG, Wong TY. Age-related macular degeneration. Lancet. 2012;379(9827):1728-38.

3. Armstrong RA, Mousavi M. Overview of Risk Factors for Age-Related Macular Degeneration (AMD). J Stem Cells. 2015;10(3):171-91.

4. Klein BE, Howard KP, lyengar SK, Sivakumaran TA, Meyers KJ, Cruickshanks KJ, et al. Sunlight exposure, pigmentation, and inciden age-related macular degeneration. Invest Ophthalmol Vis Sci. 2014; 55(9):5855-61.

5. Nowak JZ. AMD--the retinal disease with an unprecised etiopathogenesis in search of effective therapeutics. Acta Pol Pharm. 2014:71(6):900-16.

6. Fritsche LG, Fariss RN, Stambolian D, Abecasis GR, Curcio CA, Swaroop A. Age-related macular degeneration: genetics and biology coming together. Annu Rev Genomics Hum Genet. 2014;15:151-71.
7. Vempati P, Popel AS, Mac Gabhann F. Extracellular regulation of VEGF: isoforms, proteolysis, and vascular patterning. Cytokine Growth Factor Rev. 2014;25(1):1-19.

8. Koch S, Claesson-Welsh L. Signal transduction by vascular endothelia growth factor receptors. Cold Spring Harb Perspect Med. 2012;2(7):a006502.

9. Witmer AN, Vrensen GF, Van Noorden CJ, Schlingemann RO. Vascular endothelial growth factors and angiogenesis in eye disease. Prog Retin Eye Res. 2003;22(1):1-29.

10. Liu Y, Hou S, Lang W, Dai D, Wang Z, Ji X, et al. Roles of three common VEGF polymorphisms in the risk of age-related macular degeneration. Genet Test Mol Biomarkers. 2014;18(4):245-52.

11. Gupta D, Gupta V, Singh V, Prakash S, Agrawal S, Chawla S, et al. Vascular endothelial growth factor gene polymorphisms and association with age related macular degeneration in Indian patients. Meta Gene. 2016;9:249-53.

12. Stevens A, Soden J, Brenchley PE, Ralph S, Ray DW. Haplotype analysis of the polymorphic human vascular endothelial growth factor gene promoter. Cancer Res. 2003;63(4):812-6.

13. Wu X, Xin Z, Zhang W, Wu J, Chen K, Wang H, et al. Polymorphisms in the VEGFA promoter are associated with susceptibility to hepatocellular carcinoma by altering promoter activity. Int J Cancer. 2013;133(5):1085-93.

14. Janik-Papis K, Zaras M, Krzyzanowska A, Wozniak K, Blasiak J, Szaflik J, et al. Association between vascular endothelial growth factor gene polymorphisms and age-related macular degeneration in a Polish population. Exp Mol Pathol. 2009;87(3):234-8.

15. Lin JM, Wan L, Tsai YY, Lin HJ, Tsai Y, Lee CC, et al. Vascular endothelial growth factor gene polymorphisms in age-related macular degeneration. Am J Ophthalmol. 2008;145(6):1045-51.

16. Qu Y, Dai H, Zhou F, Zhang X, Xu X, Zhang X, et al. Vascular endothelial growth factor gene polymorphisms and risk of neovascular age-related macular degeneration in a Chinese cohort. Ophthalmic Res. 2011; 45(3):142-8

17. Cruz-Gonzalez F, Cabrillo Estevez L, Canete Campos C, Sanchez-Jara Sanchez A, Juan Marcos L, Gonzalez-Sarmiento R. The presence of CFH, HTRA1, ARMS2, VEGF-A and VEGF-R and the appearance of age-related macular degeneration sub-types. Arch Soc Esp Oftalmol. 2016;91(4):177-83

18. Szeto CC, Chow KM, Poon P, Szeto CY, Wong TY, Li PK. Genetic polymorphism of VEGF: Impact on longitudinal change of peritoneal transport and survival of peritoneal dialysis patients. Kidney Int. 2004:65(5):1947-55.

19. Boekhoorn SS, Isaacs A, Uitterlinden AG, van Duijn CM, Hofman A, de Jong PT, et al. Polymorphisms in the vascular endothelial growth factor gene and risk of age-related macular degeneration: the Rotterdam Study. Ophthalmology. 2008:115(11):1899-903.

20. Immonen I, Seitsonen S, Tommila P, Kangas-Kontio T, Kakko S, Savolainen ER, et al. Vascular endothelial growth factor gene variation and the response to photodynamic therapy in age-related macular degeneration. Ophthalmology. 2010;117(1):103-8.

21. Mori K, Horie-Inoue K, Gehlbach PL, Takita H, Kabasawa S, Kawasaki I, et al. Phenotype and genotype characteristics of age-related macular degeneration in a Japanese population. Ophthalmology. 2010;117(5): 928-38.

22. Kepez Yildiz B, Ozdek S, Ergun MA, Ergun S, Yaylacioglu Tuncay F, Elbeg S. CFH Y402H and VEGF Polymorphisms and Anti-VEGF Treatment Response in Exudative Age-Related Macular Degeneration. Ophthalmic Res. 2016;56(3):132-8.

23. Lu Y, Shi Y, Xue C, Yin J, Huang Z. Pooled-analysis of the associations between three polymorphisms in the VEGF gene and age-related macular degeneration. Mol Biol Rep. 2012;39(6):6547-53.

24. Kinnunen K, Yla-Herttuala S. Vascular endothelial growth factors in retinal and choroidal neovascular diseases. Ann Med. 2012:44(1):1-17.

25. Goncalves FT, Cezario SM, Calastri MC, Oliveira Cl, Souza DR, Pinhel MA, et al. Influence of VEGF-C936T genetic variant on age-related macular degeneration. Arq Bras Oftalmol. 2015;78(5):290-4.

26. Okamoto N, Tobe T, Hackett SF, Ozaki H, Vinores MA, LaRochelle W, et al. Transgenic mice with increased expression of vascular endothelial growth factor in the retina: a new model of intraretinal and subretinal neovascularization. Am J Pathol. 1997:151(1):281-91.

27. Wong CG, Taban M, Osann K, Ross-Cisneros FN, Bruice TC, Zahn G, et al. Subchoroidal Release of VEGF and bFGF Produces Choroidal Neovascularization in Rabbit. Curr Eye Res. 2017;42(2):237-43.

28. Qiu G, Stewart JM, Sadda S, Freda R, Lee S, Guven D, et al. A new model of experimental subretinal neovascularization in the rabbit. Exp Eye Res. 2006;83(1):141-52

29. Julien S, Kreppel F, Beck S, Heiduschka P, Brito V Schnichels S, et al. $A$ reproducible and quantifiable model of choroidal neovascularization induced by VEGF A165 after subretinal adenoviral gene transfer in the rabbit. Mol Vis. 2008:14:1358-72.

30. Sacilotto N, Chouliaras KM, Nikitenko LL, Lu YW, Fritzsche M, Wallace MD, et al. MEF2 transcription factors are key regulators of sprouting angiogenesis. Genes Dev. 2016;30(20):2297-309. 
31. Saint-Geniez M, Jiang A, Abend S, Liu L, Sweigard H, Connor KM, et al. $P G C$-1alpha regulates normal and pathological angiogenesis in the retina. Am J Pathol. 2013;182(1):255-65.

32. Park UC, Shin JY, Kim SJ, Shin ES, Lee JE, McCarthy LC, et al. Genetic factors associated with response to intravitreal ranibizumab in Korean patients with neovascular age-related macular degeneration. Retina 2014;34(2):288-97.

33. Ambreen F, Ismail M, Qureshi IZ. Association of gene polymorphism with serum levels of inflammatory and angiogenic factors in Pakistani patients with age-related macular degeneration. Mol Vis. 2015;21:985-99

34. Vailati FB, Crispim D, Sortica DA, Souza BM, Brondani LA, Canani LH. The $C$ allele of $-634 \mathrm{G} / \mathrm{C}$ polymorphism in the VEGFA gene is associated with increased VEGFA gene expression in human retinal tissue. Invest Ophthalmol Vis Sci. 2012;53(10):6411-5.

35. Petrovic MG, Korosec P, Kosnik M, Osredkar J, Hawlina M, Peterlin B et al. Local and genetic determinants of vascular endothelial growth factor expression in advanced proliferative diabetic retinopathy. Mol Vis. 2008;14:1382-7.

36. Hagstrom SA, Ying GS, Pauer GJ, Sturgill-Short GM, Huang J, Maguire MG, et al. VEGFA and VEGFR2 gene polymorphisms and response to anti-vascular endothelial growth factor therapy: comparison of age-related macular degeneration treatments trials (CATT). JAMA Ophthalmol. 2014;132(5):521-7.
37. Habibi I, Kort F, Sfar I, Chebil A, Bouraoui R, Ben Abdallah T, et al. Effect of Risk Alleles in CFH, C3, and VEGFA on the Response to Intravitreal Bevacizumab in Tunisian Patients with Neovascular Age-related Macular Degeneration. Klin Monbl Augenheilkd. 2016;233(4):465-70.

38. Song Y, Yang Y, Liu L, Liu X. Association between five polymorphisms in vascular endothelial growth factor gene and urinary bladder cancer risk: A systematic review and meta-analysis involving 6671 subjects. Gene. 2019;698:186-97.

39. Fang AM, Lee AY, Kulkarni M, Osborn MP, Brantley MA, Jr. Polymorphisms in the VEGFA and VEGFR-2 genes and neovascular age-related macular degeneration. Mol Vis. 2009;15:2710-9.

40. Malkiewicz A, Slominski B, Skrzypkowska M, Siebert J, Gutknecht P, Mysliwska J. The GA genotype of the $-1154 \mathrm{G} / \mathrm{A}$ (rs1570360) vascular endothelial growth factor (VEGF) is protective against hypertension-related chronic kidney disease incidence. Mol Cell Biochem. 2016:418(1-2):159-65.

41. Yadav BK, Yadav R, Chang H, Choi K, Kim JT, Park MS, et al. Genetic Polymorphisms rs699947, rs1570360, and rs3025039 on the VEGF Gene Are Correlated with Extracranial Internal Carotid Artery Stenosis and Ischemic Stroke. Ann Clin Lab Sci. 2017;47(2):144-55.

42. Boltz A, Ruiss M, Jonas JB, Tao Y, Rensch F, Weger M, et al. Role of vascular endothelial growth factor polymorphisms in the treatment success in patients with wet age-related macular degeneration. Ophthalmology. 2012;119(8):1615-20. 